Supporting Information

\title{
A Mechanism for Non-stoichiometry in the Lithium Amide/Lithium Imide Hydrogen Storage Reaction
}

\author{
William I. F. David ${ }^{\mathrm{a}, \mathrm{b}}$, Martin O. Jones ${ }^{\mathrm{b}}$, Duncan H. Gregory ${ }^{\mathrm{c}}$, Catherine M. Jewell ${ }^{\mathrm{c}}$, \\ Simon R. Johnson ${ }^{b}$, Allan Walton ${ }^{\mathrm{d}}$ and Peter P. Edwards ${ }^{\mathrm{b}}$ \\ ${ }^{a}$ ISIS Facility, Rutherford Appleton Laboratory, Chilton, Didcot, Oxon., UK OX11 0QX. \\ ${ }^{b}$ Inorganic Chemistry Laboratory, University of Oxford, South Parks Road, Oxford, UK OX3 3QR \\ ${ }^{c}$ Department of Chemistry, University of Glasgow, Glasgow G12 8QQ, UK. \\ ${ }^{d}$ Department of Metallurgy and Materials, University of Birmingham, Edgbaston, Birmingham, B15 2 TT.
}

Criteria of fit for the refinements are as follows;

Where $Y_{o, m}$ and $Y_{c, m}$ are the observed and calculated data respectively at each data point $\mathrm{m}, \mathrm{Bkg}_{\mathrm{m}}$ the background data point $\mathrm{m}, \mathrm{M}$ the number of data points, $\mathrm{P}$ the number of parameters, $\mathrm{w}_{\mathrm{m}}$ the weighting given to data point $\mathrm{m}$ which for counting statistics is given by $\mathrm{w}_{\mathrm{m}}=1 / \sigma\left(\mathrm{Y}_{\mathrm{o}, \mathrm{m}}\right)^{2}$ where $\sigma\left(\mathrm{Y}_{\mathrm{o}, \mathrm{m}}\right)$ is the error in $\mathrm{Y}_{\mathrm{o}, \mathrm{m}}$ and $\mathrm{I} \mathrm{co}_{\mathrm{o}, \mathrm{k}}$ and $\mathrm{I}_{\mathrm{c}, \mathrm{k}}$ the "observed" and calculated intensities of the $\mathrm{K}^{\text {th }}$ reflection.

$$
\begin{aligned}
& R_{p}=\sqrt{\frac{\sum\left|Y_{o, m}-Y_{c, m}\right|}{\sum Y_{o, m}}} \\
& R_{p}^{\prime}=\sqrt{\frac{\sum\left|Y_{o, m}-Y_{c, m}\right|}{\sum\left|Y_{o, m}-B k g_{m}\right|}} \\
& R_{w p}=\sqrt{\frac{\sum W_{m}\left(Y_{o, m}-Y_{c, m}\right)^{2}}{\sum W_{m} Y_{o, m}^{2}}} \\
& R_{w p}^{\prime}=\sqrt{\frac{\sum W_{m}\left(Y_{o, m}-Y_{c, m}\right)^{2}}{\sum W_{m}\left(Y_{o, m}{ }^{2}-B k g_{m}\right)}} \\
& R_{\exp }=\sqrt{\frac{\sum M-P}{\sum W_{m} Y_{o, m}^{2}}} \\
& R_{\exp }^{\prime}=\sqrt{\frac{\sum M-P}{\sum W_{m}\left(Y_{o, m}-B k g_{m}\right)^{2}}} \\
& \text { Goodness of fit, GOF }=\mathrm{chi}^{2}=\frac{R_{w p}}{R_{\exp }}=\sqrt{\frac{\sum\left|I_{"^{\prime \prime} k}-I_{c, k}\right|}{\sum I_{"^{\prime \prime}, k}}} \\
& \text { "Durbin-Watson", } d=\frac{\sum_{m=2}^{M}\left(\Delta Y_{m}-\Delta Y_{m-1}\right)}{\sum_{m=1}^{M}\left(\Delta Y_{m}\right)^{2}} ; \quad \Delta Y_{m}=Y_{o, m}-Y_{c, m}
\end{aligned}
$$




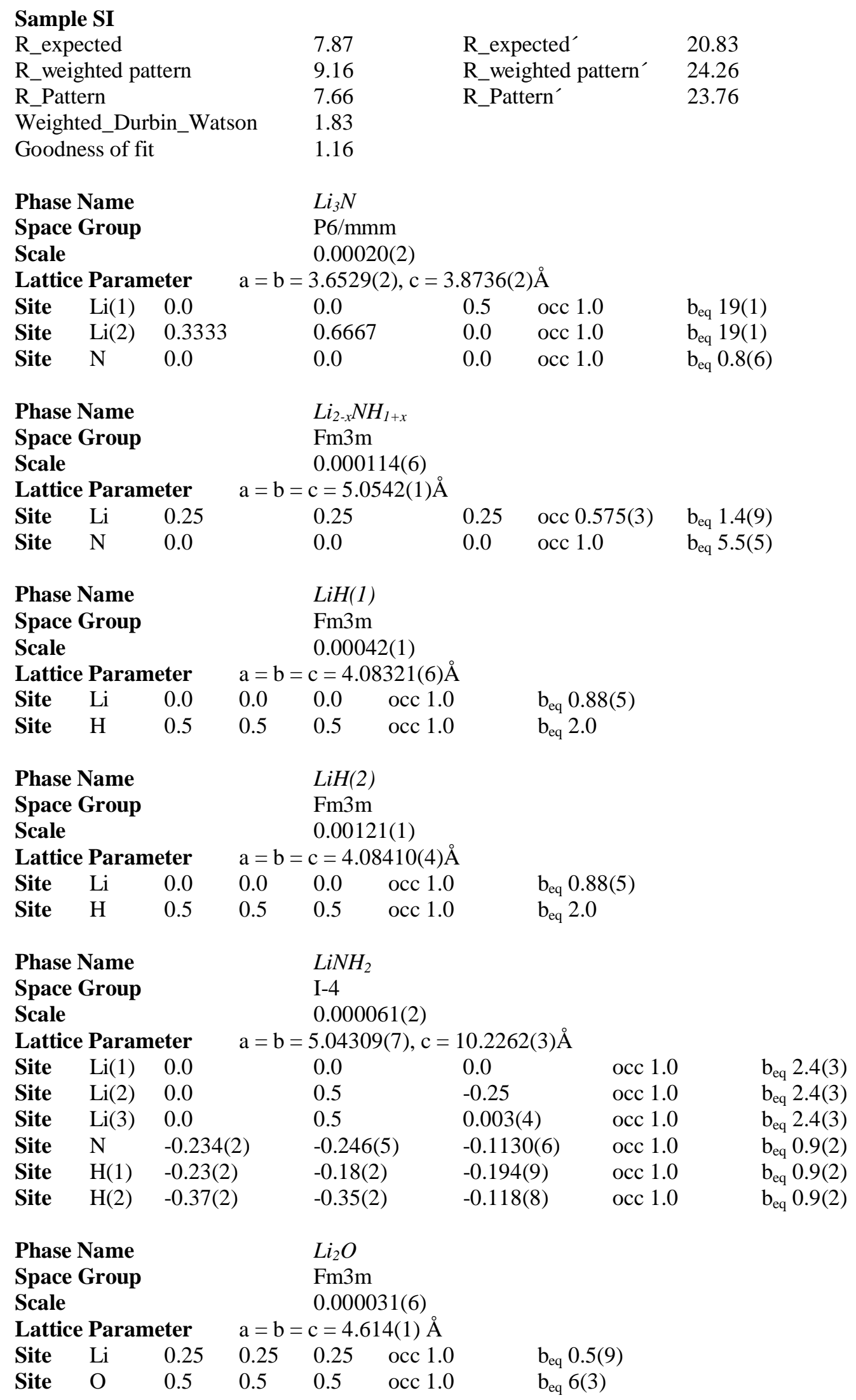


Sample SII

$\begin{array}{lclr}\text { R_expected } & 7.45 & \text { R_expected' } & 20.95 \\ \text { R_weighted pattern } & 8.62 & \text { R_weighted pattern' }^{\prime} & 24.23 \\ \text { R_Pattern } & 7.24 & \text { R_Pattern' } & 23.14 \\ \text { Weighted_Durbin_Watson } & 1.80 & & \\ \text { Goodness of fit } & 1.16 & & \\ & & \\ \text { Li-N-H-00 - Li-N-H-10 Lattice Parameter } & \text { a_hi }=5.07919(60) \AA \\ \text { Li-N-H-00 - Li-N-H-10 Lattice Parameter } & \text { a_lo }=5.00013(7) \AA \\ \text { Li-N-H-00 - Li-N-H-10 Li Occ. Parameter } & \text { xLi_hi }=0.828(12) \\ \text { Li-N-H-00 - Li-N-H-10 Li Occ.Parameter } & \text { xLi_lo }=0.508(20)\end{array}$

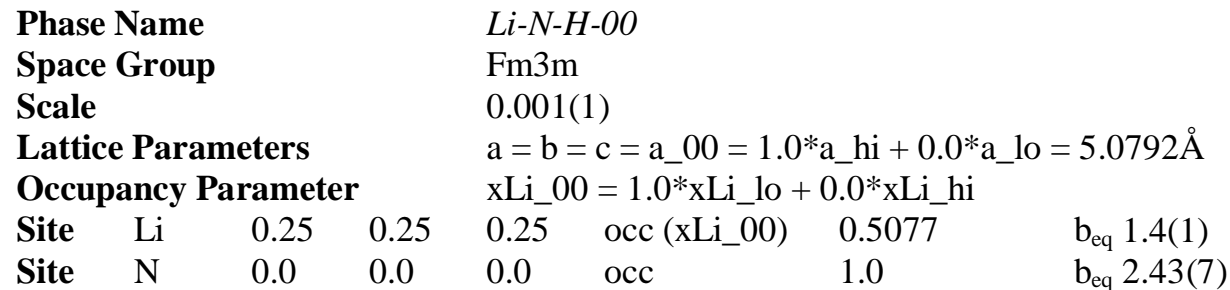

Phase Name $\quad \mathrm{Li}-\mathrm{N}-\mathrm{H}-\mathrm{O} 1$

Space Group $\quad$ Fm3m

Scale $0.030(8)$

Lattice Parameter $\quad \mathrm{a}=\mathrm{b}=\mathrm{c}=\mathrm{a} \_01=0.9 * \mathrm{a} \_\mathrm{hi}+0.1 * \mathrm{a} \_$lo $=5.0713 \AA$

Occupancy Parameter $\quad$ xLi_01 $=0.9 * x L i \_l o+0.1 * x L i \_h i$

$\begin{array}{llllllll}\text { Site } & \mathrm{Li} & 0.25 & 0.25 & 0.25 & \text { occ }\left(x L i \_01\right) & 0.5396 & \mathrm{~b}_{\text {eq }} 1.4(1) \\ \text { Site } & \mathrm{N} & 0.0 & 0.0 & 0.0 & \text { occ } & 1.0 & \mathrm{~b}_{\text {eq }} 2.43(7)\end{array}$

Phase Name

$\mathrm{Li}-\mathrm{N}-\mathrm{H}-\mathrm{O} 2$

Space Group Fm3m

Scale

Lattice Parameter

$0.163(9)$

Occupancy Parameter

$\begin{array}{llll}\text { Site } & \mathrm{Li} & 0.25 & 0.25\end{array}$

Site $\quad \mathrm{N} \quad 0.0 \quad 0.0$

Phase Name

$\mathrm{a}=\mathrm{b}=\mathrm{c}=\mathrm{a} \_01=0.8 * \mathrm{a} \_$hi $+0.2 * \mathrm{a} \_\mathrm{lo}=5.0634 \AA$

xLi_02 $=0.8 * x L i \_l o+0.2 * x L i \_h i$

$0.25 \quad$ occ (xLi_02) $0.5716 \quad \mathrm{~b}_{\mathrm{eq}} 1.4(1)$

$\begin{array}{llll}0.0 & \text { occ } & 1.0 & b_{\text {eq }} 2.43(7)\end{array}$

\begin{tabular}{|c|c|c|c|c|c|c|c|}
\hline Phas & VaI & & & $L i-N$ & -03 & & \\
\hline Spac & Gro & & & $\mathrm{Fm} 31$ & & & \\
\hline Scal & & & & 0.17 & & & \\
\hline Latt & $\begin{array}{l}\mathrm{Pa} \\
\text { anc }\end{array}$ & eter & & $\begin{array}{l}a=b \\
x L i\end{array}$ & $\begin{array}{l}c=a \_01=0.7 \\
=0.7 * x L i 10\end{array}$ & a_hi +0. & $=5.0555 \AA$ \\
\hline Site & $\mathrm{Li}$ & 0.25 & 0.25 & 0.25 & occ (xLi_03) & 0.6036 & $\mathrm{~b}_{\mathrm{eq}} 1.4(1)$ \\
\hline Site & $\mathrm{N}$ & 0.0 & 0.0 & 0.0 & occ & 1.0 & $b_{\text {eq }} 2.43(7)$ \\
\hline
\end{tabular}

Phase Name

$\mathrm{Li}-\mathrm{N}-\mathrm{H}-04$

Space Group Fm3m

Scale $0.239(9)$

Lattice Parameter $\quad \mathrm{a}=\mathrm{b}=\mathrm{c}=\mathrm{a} \_01=0.6 * \mathrm{a} \_\mathrm{hi}+0.4 * \mathrm{a} \_$lo $=5.0476 \AA$

Occupancy Parameter $\quad$ xLi_04 $=0.6^{*} \times \mathrm{LLi}$ _lo $+0.4^{*} \times \mathrm{xi}$ hi

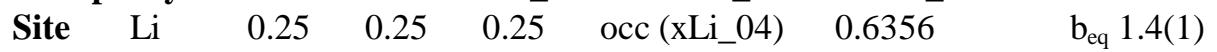

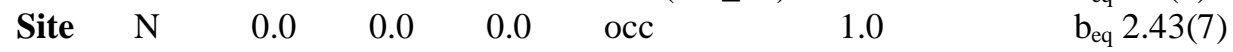

Phase Name Li-N-H-O5

Space Group Fm3m 


\begin{tabular}{|c|c|c|c|c|c|c|c|}
\hline \multirow{3}{*}{\multicolumn{4}{|c|}{$\begin{array}{l}\text { Scale } \\
\text { Lattice Parameter } \\
\text { Occupancy Parameter }\end{array}$}} & \multicolumn{4}{|c|}{$0.239(9)$} \\
\hline & & & & \multirow{2}{*}{\multicolumn{4}{|c|}{$\begin{array}{l}\mathrm{a}=\mathrm{b}=\mathrm{c}=\mathrm{a} \_01=0.5^{*} \mathrm{a} \_\mathrm{hi}+0.5^{*} \mathrm{a} \_l \mathrm{lo}=5.0397 \AA \\
\mathrm{xLi} 05=0.5^{*} \mathrm{xLi} \_ \text {lo }+0.5^{*} \mathrm{xLi} \text { hi }\end{array}$}} \\
\hline & & & & & & & \\
\hline & $\mathrm{Li}$ & 0.25 & 0.25 & 0.25 & occ (xLi_05) & 0.6675 & $\mathrm{~b}_{\mathrm{eq}} 1.4(1)$ \\
\hline Site & $\mathrm{N}$ & 0.0 & 0.0 & 0.0 & occ & 1.0 & $b_{\text {eq }} 2.43(7)$ \\
\hline \multicolumn{4}{|c|}{ Phase Name } & \multicolumn{4}{|c|}{$\mathrm{Li}-\mathrm{N}-\mathrm{H}-06$} \\
\hline \multicolumn{4}{|c|}{ Space Group } & \multicolumn{4}{|c|}{ Fm3m } \\
\hline \multicolumn{4}{|c|}{ Scale } & \multicolumn{4}{|c|}{$0.285(9)$} \\
\hline \multicolumn{4}{|c|}{ Lattice Parameter } & \multicolumn{4}{|c|}{$\mathrm{a}=\mathrm{b}=\mathrm{c}=\mathrm{a} \_01=0.4 * \mathrm{a} \_$hi $+0.6 * \mathrm{a} \_\mathrm{lo}=5.0318 \AA$} \\
\hline \multicolumn{4}{|c|}{ Occupancy Parameter } & \multicolumn{4}{|c|}{ xLi_06 = 0.4*xLi_lo + 0.6*xLi_hi } \\
\hline Site & $\mathrm{Li}$ & 0.25 & 0.25 & $0.2 \overline{5}$ & occ (xLi_06) & 0.6995 & $\mathrm{~b}_{\mathrm{eq}} 1.4(1)$ \\
\hline Site & $\mathrm{N}$ & 0.0 & 0.0 & 0.0 & occ & 1.0 & $b_{\text {eq }} 2.43(7)$ \\
\hline \multicolumn{4}{|c|}{ Phase Name } & \multicolumn{4}{|c|}{$\mathrm{Li}-\mathrm{N}-\mathrm{H}-\mathrm{O} 7$} \\
\hline \multicolumn{4}{|c|}{ Space Group } & \multicolumn{4}{|c|}{ Fm3m } \\
\hline \multicolumn{4}{|c|}{ Scale } & \multicolumn{4}{|c|}{$0.333(10)$} \\
\hline \multirow{2}{*}{\multicolumn{4}{|c|}{$\begin{array}{l}\text { Lattice Parameter } \\
\text { Occupancy Parameter }\end{array}$}} & \multirow{2}{*}{\multicolumn{4}{|c|}{$\begin{array}{l}\mathrm{a}=\mathrm{b}=\mathrm{c}=\mathrm{a} \_01=0.3 * \mathrm{a} \_ \text {hi }+0.7 * \mathrm{a} \_l \mathrm{lo}=5.0239 \AA \\
\mathrm{xLi} 07=0.3 * x \mathrm{xL} \text { lo }+0.7 * x \mathrm{xi} \text { hi }\end{array}$}} \\
\hline & & & & & & & \\
\hline Site & $\mathrm{Li}$ & 0.25 & 0.25 & 0.25 & occ (xLi_07) & 0.7315 & $\mathrm{~b}_{\mathrm{eq}} 1.4(1)$ \\
\hline Site & $\mathrm{N}$ & 0.0 & 0.0 & 0.0 & occ & 1.0 & $b_{\text {eq }} 2.43(7)$ \\
\hline Pha & Nan & & & $L i-N$ & -08 & & \\
\hline Spa & Gro & & & Fm3r & & & \\
\hline Scal & & & & 0.438 & & & \\
\hline Latt & $\mathbf{P a}$ & eter & & $a=b$ & $c=a \_01=0.2$ & a_hi +0 . & $=5.0159 \AA$ \\
\hline Occ & ancy & rame & & xLi_ & $=0.2 * x \mathrm{Li} \_l \mathrm{lo}$ & $0.8 * x \mathrm{Li}_{-}$ & \\
\hline Site & $\mathrm{Li}$ & 0.25 & 0.25 & 0.25 & occ (xLi_08) & 0.7635 & $\mathrm{~b}_{\mathrm{eq}} 1.4(1)$ \\
\hline Site & $\mathrm{N}$ & 0.0 & 0.0 & 0.0 & occ & 1.0 & $b_{\text {eq }} 2.43(7)$ \\
\hline Pha & Nan & & & $L i-N$ & -09 & & \\
\hline Spa & Gro & & & Fm3r & & & \\
\hline Scal & & & & 0.738 & & & \\
\hline Latt & $\mathbf{P a}$ & ter & & $\mathrm{a}=\mathrm{b}$ & $c=a \_01=0.1$ & a_hi +0 . & $=5.0080 \AA$ \\
\hline Occ & ancs & rame & & xLi_ & $=0.1 * x L i \_l o+$ & $0.9 * x \mathrm{Li}_{-}$ & \\
\hline Site & $\mathrm{Li}$ & 0.25 & 0.25 & 0.25 & occ (xLi_09) & 0.7955 & $\mathrm{~b}_{\mathrm{eq}} 1.4(1)$ \\
\hline Site & $\mathrm{N}$ & 0.0 & 0.0 & 0.0 & occ & 1.0 & $\mathrm{~b}_{\mathrm{eq}} 2.43(7)$ \\
\hline Pha & Nan & & & $L i-N$ & -10 & & \\
\hline Spa & Gro & & & $\mathrm{Fm} 31$ & & & \\
\hline Scal & & & & 1.53 & & & \\
\hline Latt & $\mathbf{P a}$ & eter & & $a=b$ & $c=a \_01=0.0$ & a_hi +1 . & $=5.00013 \AA$ \\
\hline Occ & ancs & rame & & $\mathrm{xLi}_{-}$ & $=0.0 * x L i \_l o+$ & $1.0 * x \mathrm{Li}_{-}$ & \\
\hline Site & $\mathrm{Li}$ & 0.25 & 0.25 & 0.25 & occ (xLi_10) & 0.8274 & $\mathrm{~b}_{\mathrm{eq}} 1.4(1)$ \\
\hline Site & $\mathrm{N}$ & 0.0 & 0.0 & 0.0 & occ & 1.0 & $b_{\text {eq }} 2.43(7)$ \\
\hline Pha & Nan & & & $\mathrm{LiH}$ & & & \\
\hline Spa & Gro & & & Fm3r & & & \\
\hline Scal & & & & 0.001 & 2(2) & & \\
\hline Latt & $\mathbf{P a}$ & eter & & $a=b$ & $c=4.08321(2)$ & & \\
\hline Site & $\mathrm{Li}$ & 0.0 & 0.0 & 0.0 & occ 1.0 & $b_{\text {eq }} 0.92$ & \\
\hline Site & $\mathrm{H}$ & 0.5 & 0.5 & 0.5 & occ 1.0 & $\mathrm{~b}_{\mathrm{eq}} 2.0$ & \\
\hline Pha & Nan & & & $\mathrm{Li}_{2} \mathrm{O}$ & & & \\
\hline Spa & Gro & & & Fm3r & & & \\
\hline
\end{tabular}


Scale $\quad 0.000049(2)$

Lattice Parameter $\quad \mathrm{a}=\mathrm{b}=\mathrm{c}=4.6124(1) \AA$

$\begin{array}{llllll}\text { Site } & \mathrm{Li} & 0.25 & 0.25 & 0.25 & \text { occ } 1.0\end{array}$

$\begin{array}{llllll}\text { Site } & O & 0.5 & 0.5 & 0.5 & \text { occ } 1.0\end{array}$

$b_{\text {eq }} 1.8(3)$

$b_{\text {eq }} 1.1(2)$ 
Sample SIII

$\begin{array}{llll}\text { R_expected } & 7.79 & \text { R_expected' } & 18.25 \\ \text { R_weighted pattern } & 9.43 & \text { R_weighted pattern' } & 22.10 \\ \text { R_Pattern } & 7.94 & \text { R_Pattern' } & 24.01\end{array}$

Weighted_Durbin_Watson 1.75

Goodness of fit $\quad 1.21$

Li-N-H-00 - Li-N-H-10 Lattice Parameter

a_hi $=5.06992(40) \AA$

Li-N-H-00 - Li-N-H-10 Lattice Parameter

a_lo $=4.99957(6) \AA$

Li-N-H-00 - Li-N-H-10 Li Occ. Parameter

xLi_hi $=0.850(6)$

Li-N-H-00 - Li-N-H-10 Li Occ. Parameter

xLi_lo $=0.590(10)$

\begin{tabular}{|c|c|c|c|c|c|c|c|}
\hline \multicolumn{4}{|c|}{ Phase Name } & \multicolumn{4}{|c|}{$\mathrm{Li}-\mathrm{N}-\mathrm{H}-\mathrm{OO}$} \\
\hline \multicolumn{4}{|c|}{ Space Group } & \multicolumn{4}{|c|}{ Fm3m } \\
\hline \multicolumn{4}{|c|}{ Scale } & \multicolumn{4}{|c|}{$0.153(12)$} \\
\hline \multicolumn{4}{|c|}{ Lattice Parameter } & \multicolumn{4}{|c|}{$\mathrm{a}=\mathrm{b}=\mathrm{c}=\mathrm{a} \_00=1.0 * \mathrm{a} \_\mathrm{hi}+0.0 * \mathrm{a} \_\mathrm{lo}=5.0699 \AA$} \\
\hline \multicolumn{4}{|c|}{ Occupancy Parameter } & \multicolumn{4}{|c|}{ xLi_00 $=1.0 * x L i \_l o+0.0 * x L i$ hi } \\
\hline Site & $\mathrm{Li}$ & 0.25 & 0.25 & $0.2 \overline{5}$ & occ (xLi_00) & 0.5894 & $b_{\text {eq }} 1.79(8)$ \\
\hline Site & $\mathrm{N}$ & 0.0 & 0.0 & 0.0 & occ -1 & 1.0 & $b_{\text {eq }} 2.43(4)$ \\
\hline \multicolumn{4}{|c|}{ Phase Name } & \multicolumn{4}{|c|}{$\mathrm{Li}-\mathrm{N}-\mathrm{H}-\mathrm{Ol}$} \\
\hline \multicolumn{4}{|c|}{ Space Group } & \multicolumn{4}{|c|}{ Fm3m } \\
\hline \multicolumn{4}{|c|}{ Scale } & \multicolumn{4}{|c|}{$0.245(12)$} \\
\hline \multirow{2}{*}{\multicolumn{4}{|c|}{$\begin{array}{l}\text { Lattice Parameter } \\
\text { Occupancy Parameter }\end{array}$}} & \multirow{2}{*}{\multicolumn{4}{|c|}{$\begin{array}{l}\mathrm{a}=\mathrm{b}=\mathrm{c}=\mathrm{a} \_01=0.9 * \mathrm{a} \_\mathrm{hi}+0.1 * \mathrm{a} \_\mathrm{lo}=5.0628 \AA \\
\mathrm{xLi} 01=0.9 * x \mathrm{xi} \_l o+0.1 * x \mathrm{Li} \_\mathrm{hi}\end{array}$}} \\
\hline & & & & & & & \\
\hline Site & $\mathrm{Li}$ & 0.25 & 0.25 & $0.2 \overline{5}$ & occ (xLi_01) & 0.6156 & $b_{e q} 1.79(8)$ \\
\hline Site & $\mathrm{N}$ & 0.0 & 0.0 & 0.0 & occ & 1.0 & $b_{\text {eq }} 2.43(4)$ \\
\hline \multicolumn{4}{|c|}{ Phase Name } & \multicolumn{4}{|c|}{$\mathrm{Li}-\mathrm{N}-\mathrm{H}-\mathrm{O} 2$} \\
\hline \multicolumn{4}{|c|}{ Space Group } & \multicolumn{4}{|c|}{ Fm3m } \\
\hline \multicolumn{4}{|c|}{ Scale } & \multicolumn{4}{|c|}{$0.400(15)$} \\
\hline \multirow{2}{*}{\multicolumn{4}{|c|}{$\begin{array}{l}\text { Lattice Parameter } \\
\text { Occupancy Parameter }\end{array}$}} & \multirow{2}{*}{\multicolumn{4}{|c|}{ 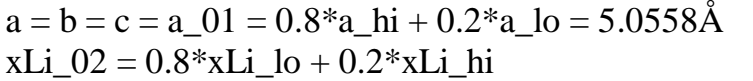 }} \\
\hline & & & & & & & \\
\hline Site & $\mathrm{Li}$ & 0.25 & 0.25 & 0.25 & occ (xLi_02) & 0.6417 & $b_{\text {eq }} 1.79(8)$ \\
\hline Site & $\mathrm{N}$ & 0.0 & 0.0 & 0.0 & occ & 1.0 & $b_{\text {eq }} 2.43(4)$ \\
\hline \multicolumn{4}{|c|}{ Phase Name } & $L i-N$ & -03 & & \\
\hline Spac & Gro & & & Fm3 & & & \\
\hline Scal & & & & $0.45^{\circ}$ & & & \\
\hline Latt & $\mathbf{P a}$ & eter & & $a=b$ & $c=a \_01=0.7$ & A_hi +0. & $=5.0488 \AA$ \\
\hline Occ & anc & rame & & $x \mathrm{Li}_{-}$ & $=0.7 * x L i \_l o+$ & $.3 * \times \mathrm{Li}_{-}$ & \\
\hline Site & $\mathrm{Li}$ & 0.25 & 0.25 & 0.25 & occ (xLi_03) & 0.6678 & $b_{\text {eq }} 1.79(8)$ \\
\hline Site & $\mathrm{N}$ & 0.0 & 0.0 & 0.0 & occ & 1.0 & $b_{\text {eq }} 2.43(4)$ \\
\hline Phas & NaI & & & $L i-N$ & -04 & & \\
\hline Spac & Gro & & & Fm31 & & & \\
\hline Scal & & & & $0.57^{\prime}$ & & & \\
\hline Latt & $\mathbf{P a}$ & eter & & $a=b$ & $c=a \_01=0.6$ & a_hi +0. & $=5.0418 \AA$ \\
\hline Occ & anc & rame & & xLi_ & $=0.6 * x L i \_l o+$ & $.4 * x \mathrm{Li}_{-}$ & \\
\hline Site & $\mathrm{Li}$ & 0.25 & 0.25 & 0.25 & occ (xLi_04) & 0.6940 & $\mathrm{~b}_{\mathrm{eq}} 1.79(8)$ \\
\hline Site & $\mathrm{N}$ & 0.0 & 0.0 & 0.0 & occ & 1.0 & $b_{\text {eq }} 2.43(4)$ \\
\hline Phas & & & & $L i-N$ & & & \\
\hline Spac & Gro & & & Fm3 & & & \\
\hline
\end{tabular}




\begin{tabular}{|c|c|c|c|c|c|c|c|}
\hline \multicolumn{4}{|c|}{$\begin{array}{l}\text { Scale } \\
\text { Lattice Parameter } \\
\text { Occupancy Parameter }\end{array}$} & \multicolumn{4}{|c|}{$\begin{array}{l}0.626(15) \\
\mathrm{a}=\mathrm{b}=\mathrm{c}=\mathrm{a} \_01=0.5^{*} \mathrm{a} \_\mathrm{hi}+0.5^{*} \mathrm{a} \_l \mathrm{lo}=5.0347 \AA \\
\mathrm{xLi} \_05=0.5^{*} \mathrm{xLi} \text { _lo }+0.5^{*} \mathrm{xLi} \text { hi }\end{array}$} \\
\hline Site & $\mathrm{Li}$ & 0.25 & 0.25 & 0.25 & occ (xLi_05) & 0.7201 & $\mathrm{~b}_{\mathrm{eq}} 1.79(8)$ \\
\hline Site & $\mathrm{N}$ & 0.0 & 0.0 & 0.0 & occ & 1.0 & $b_{\text {eq }} 2.43(4)$ \\
\hline \multicolumn{4}{|c|}{ Phase Name } & \multicolumn{4}{|c|}{$\mathrm{Li}-\mathrm{N}-\mathrm{H}-06$} \\
\hline \multicolumn{4}{|c|}{ Space Group } & \multicolumn{4}{|c|}{$\mathrm{Fm} 3 \mathrm{~m}$} \\
\hline \multicolumn{4}{|c|}{ Scale } & \multicolumn{4}{|c|}{$0.756(18)$} \\
\hline \multicolumn{4}{|c|}{ Lattice Parameter } & \multicolumn{4}{|c|}{$\mathrm{a}=\mathrm{b}=\mathrm{c}=\mathrm{a} \_01=0.4 * \mathrm{a} \_\mathrm{hi}+0.6^{*} \mathrm{a} \_\mathrm{lo}=5.0277 \AA$} \\
\hline \multicolumn{4}{|c|}{ Occupancy Parameter } & \multicolumn{4}{|c|}{ xLi_06 = 0.4*xLi_lo + 0.6*xLi_hi } \\
\hline Site & $\mathrm{Li}$ & 0.25 & 0.25 & 0.25 & occ (xLi_06) & 0.7463 & $b_{\text {eq }} 1.79(8)$ \\
\hline Site & $\mathrm{N}$ & 0.0 & 0.0 & 0.0 & occ & 1.0 & $b_{\text {eq }} 2.43(4)$ \\
\hline \multicolumn{4}{|c|}{ Phase Name } & \multicolumn{4}{|c|}{$\mathrm{Li}-\mathrm{N}-\mathrm{H}-\mathrm{O} 7$} \\
\hline \multicolumn{4}{|c|}{ Space Group } & \multicolumn{4}{|c|}{ Fm3m } \\
\hline \multicolumn{4}{|c|}{ Scale } & \multicolumn{4}{|c|}{$0.874(20)$} \\
\hline \multirow{2}{*}{\multicolumn{4}{|c|}{$\begin{array}{l}\text { Lattice Parameter } \\
\text { Occupancy Parameter }\end{array}$}} & \multirow{2}{*}{\multicolumn{4}{|c|}{$\begin{array}{l}\mathrm{a}=\mathrm{b}=\mathrm{c}=\mathrm{a} \_01=0.3 * \mathrm{a} \_\mathrm{hi}+0.7 * \mathrm{a} \_\mathrm{lo}=5.0207 \AA \\
\mathrm{xLi} \_07=0.3 * x \mathrm{xi} \_l \mathrm{l}+0.7 * \mathrm{xLi} \text { hi }\end{array}$}} \\
\hline & & & & & & & \\
\hline Site & $\mathrm{Li}$ & 0.25 & 0.25 & 0.25 & occ (xLi_07) & 0.7724 & $b_{\text {eq }} 1.79(8)$ \\
\hline Site & $\mathrm{N}$ & 0.0 & 0.0 & 0.0 & occ & 1.0 & $b_{\text {eq }} 2.43(4)$ \\
\hline \multicolumn{4}{|c|}{ Phase Name } & \multicolumn{4}{|c|}{$\mathrm{Li}-\mathrm{N}-\mathrm{H}-08$} \\
\hline Spac & Gro & & & Fm3r & & & \\
\hline Scale & & & & 1.251 & & & \\
\hline Latti & $\mathbf{P a}$ & eter & & $\mathrm{a}=\mathrm{b}$ & $c=a \_01=0.2$ & a_hi +0.8 & $=5.0136 \AA$ \\
\hline Occu & anc & rame & & xLi_ & $=0.2 * x \mathrm{Li} \_l \mathrm{lo}+$ & $0.8 * x \mathrm{xi} \_$ & \\
\hline Site & $\mathrm{Li}$ & 0.25 & 0.25 & 0.25 & occ (xLi_08) & 0.7985 & $b_{\text {eq }} 1.79(8)$ \\
\hline Site & $\mathrm{N}$ & 0.0 & 0.0 & 0.0 & occ & 1.0 & $b_{\text {eq }} 2.43(4)$ \\
\hline Phas & Nan & & & $L i-N-$ & -09 & & \\
\hline Spac & Gro & & & Fm3r & & & \\
\hline Scale & & & & $2.04 \mathrm{C}$ & & & \\
\hline Latti & $\mathbf{P a}$ & eter & & $\mathrm{a}=\mathrm{b}$ & $c=a \_01=0.1$ & a_hi +0.9 & $=5.0066 \AA$ \\
\hline Occu & anc & rame & & xLi_( & $=0.1 * x{ }^{2}{ }_{1} l o+$ & $0.9 * x L i \_1$ & \\
\hline Site & $\mathrm{Li}$ & 0.25 & 0.25 & 0.25 & occ (xLi_09) & 0.8247 & $b_{\text {eq }} 1.79(8)$ \\
\hline Site & $\mathrm{N}$ & 0.0 & 0.0 & 0.0 & occ & 1.0 & $b_{\text {eq }} 2.43(4)$ \\
\hline Phas & Nan & & & $L i-N$ & -10 & & \\
\hline Spac & Gro & & & Fm3r & & & \\
\hline Scale & & & & 4.186 & & & \\
\hline Latti & $\mathbf{P a}$ & eter & & $a=b$ & $c=a \_01=0.0$ & a_hi + $1 .($ & $=4.9996 \AA$ \\
\hline Occu & inc & rame & & $\mathrm{xLi}_{-}$ & $=0.0 * x \mathrm{Li} \_l \mathrm{lo}+$ & $1.0 * x \mathrm{xi} \_$ & \\
\hline Site & $\mathrm{Li}$ & 0.25 & 0.25 & 0.25 & occ (xLi_10) & 0.8508 & $b_{\text {eq }} 1.79(8)$ \\
\hline Site & $\mathrm{N}$ & 0.0 & 0.0 & 0.0 & occ & 1.0 & $b_{\text {eq }} 2.43(4)$ \\
\hline Phas & Van & & & $\mathrm{LiH}$ & & & \\
\hline Spac & Gro & & & Fm3r & & & \\
\hline Scale & & & & 0.002 & 2(3) & & \\
\hline Latti & $\mathbf{P a}$ & eter & $a=b$ & $c=4$. & $32(1) \AA$ & & \\
\hline Site & $\mathrm{Li}$ & 0.0 & 0.0 & 0.0 & occ 1.0 & $b_{\text {eq }} 1.02$ & \\
\hline Site & $\mathrm{H}$ & 0.5 & 0.5 & 0.5 & occ 1.0 & $\mathrm{~b}_{\text {eq }} 1.8(1$ & \\
\hline Phas & & & & $\mathrm{Li}_{2} \mathrm{O}$ & & & \\
\hline Spac & Gro & & & Fm3r & & & \\
\hline
\end{tabular}




\begin{tabular}{lllllll}
\multicolumn{2}{l}{ Scale } & \multicolumn{3}{l}{$0.000196(4)$} \\
\multicolumn{2}{l}{ Lattice Parameter } & \multicolumn{4}{l}{$\mathrm{a}=\mathrm{b}=\mathrm{c}=4.6128(4) \AA \AA$} \\
Site & $\mathrm{Li}$ & 0.25 & 0.25 & 0.25 & occ 1.0 & $\mathrm{~b}_{\mathrm{eq}} 2.5(2)$ \\
Site & $\mathrm{O}$ & 0.5 & 0.5 & 0.5 & occ 1.0 & $\mathrm{~b}_{\mathrm{eq}} 1.0(1)$
\end{tabular}


Supporting Information Figures

Figure 1 The observed (dotted line), calculated (solid line) and difference plots $\left(\mathrm{Y}_{\mathrm{obs}}-\mathrm{Y}_{\text {calc }}\right.$, solid line in box) for the structure refinement of sample SII from X-ray synchrotron diffraction data collected at $\lambda=0.8022 \AA$ and with a step size of $0.003 \AA$. The Bragg peak positions for $\mathrm{LiH}$, all $\mathrm{Li}_{2} \mathrm{NH}$ phases and $\mathrm{Li}_{2} \mathrm{O}$ are all shown by the vertical tick marks The complex microstructure and two-theta dependent strain broadening in these data can be observed on the low 2-theta sides of the $\mathrm{Li}_{2} \mathrm{NH}$ diffraction peaks.

Figure 2 The observed (dotted line), calculated (solid line) and difference plots ( $\mathrm{Y}_{\mathrm{obs}}-\mathrm{Y}_{\text {calc }}$, solid line in box) for the structure refinement of sample SIII from X-ray synchrotron diffraction data collected at $\lambda=0.8022 \AA$ and with a step size of $0.003 \AA$. The Bragg peak positions for $\mathrm{LiH}$, all $\mathrm{Li}_{2} \mathrm{NH}$ phases and $\mathrm{Li}_{2} \mathrm{O}$ are all shown by the vertical tick marks The complex microstructure and two-theta dependent strain broadening in these data can be observed on the low 2-theta sides of the $\mathrm{Li}_{2} \mathrm{NH}$ diffraction peaks. 


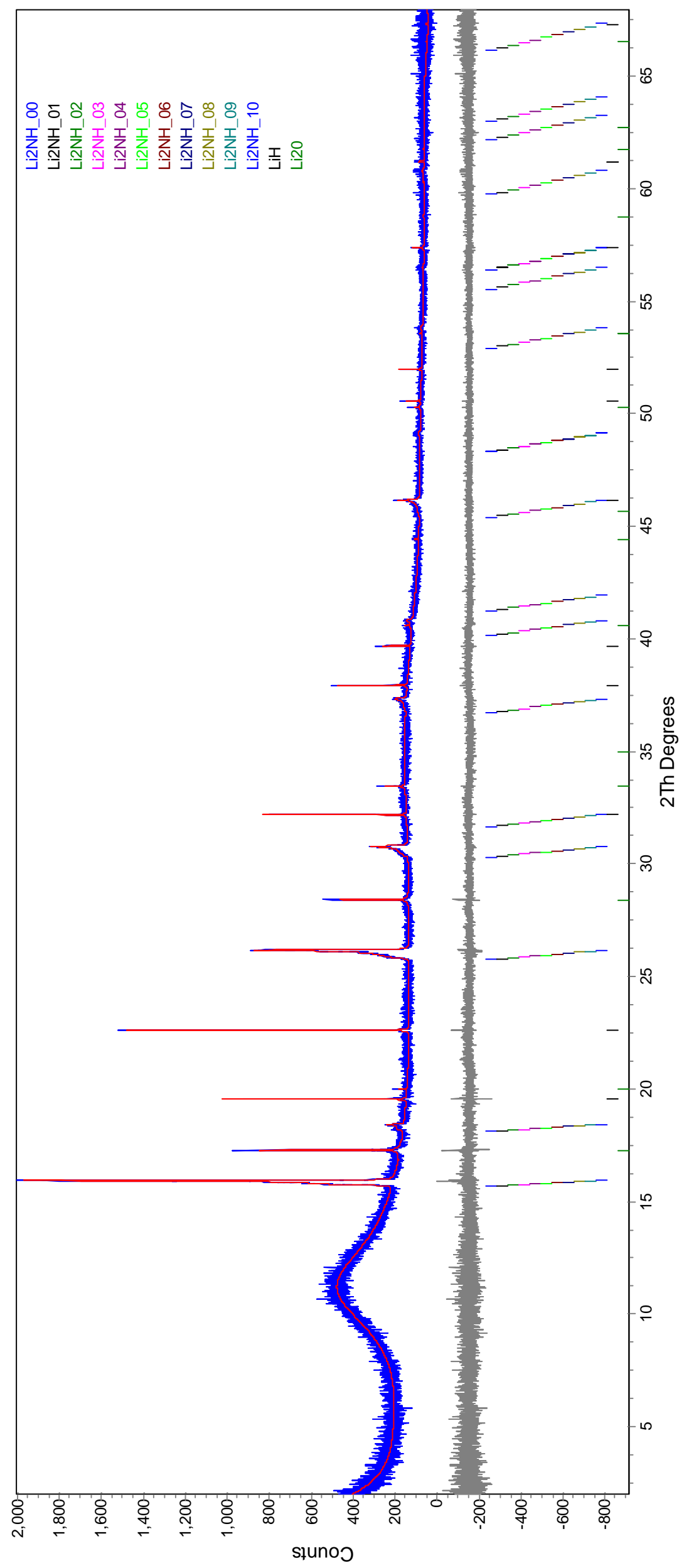

$\frac{\circ}{5}$ 


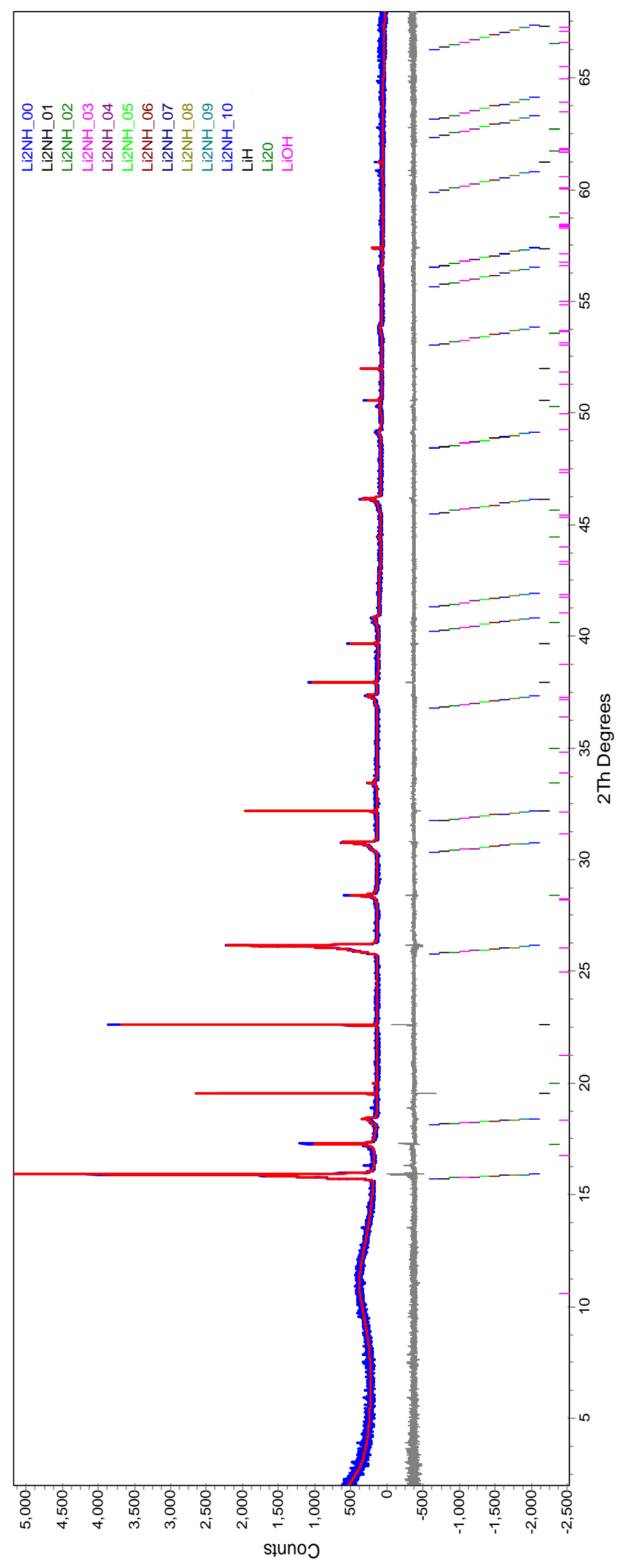

$\bar{\sigma}$ 